Estudios Constitucionales, Año 15, No 1, 2017, pp. 193 - 216

ISSN 07180195

Centro de Estudios Constitucionales de Chile Universidad de Talca

"Los derechos culturales y su gestión en Honduras"

\title{
LOS DERECHOS CULTURALES Y SU GESTIÓN EN HONDURAS*
}

\section{THE CULTURAL RIGHTS, AND THEIR MANAGEMENT IN HONDURAS}

\author{
Claudia María Castro Valle** \\ Universidad Tecnológica Centroamericana, Honduras \\ claudia.castro@unitec.edu
}

RESUMEN: Desde hace varias décadas los derechos culturales han sido una preocupación de la sociedad internacional que ha quedado plasmada en una diversidad de instrumentos internacionales, algunos de los cuales han sido suscritos y ratificados por Honduras. Las obligaciones adquiridas se han traspuesto en el ordenamiento jurídico nacional por medio de instrumentos que le han ido dando forma a la institucionalidad actual, siendo -tal vez-el más notable la Constitución de la República de Honduras.

Es relevante determinar si el marco normativo-institucional que llena de contenido las categorías que corresponden a los derechos relativos a la cultura que como ciudadanos tenemos, es adecuado para ir respondiendo a las obligaciones internacionales adquiridas por Honduras. Igual importancia tiene entender los esfuerzos del gobierno de la República para gestionar adecuadamente sus recursos e instituciones de tal forma que el derecho a la cultura quede plenamente garantizado.

ABSTRACT: For decades, cultural rights have been a concern of the international society that has been reflected in a variety of international instruments, some of which have been signed and ratified by Honduras. These international obligations have been transposed into the national law by instruments that have shaped the current institutional framework, being perhaps the most significant, the Constitution of the Republic of Honduras.

It is irrelevant to understand whether actual policy and institutional framework that fill with content the categories that correspond to the rights relating to culture that we are granted as citizens, are appropriate to fulfill the international obligations undertaken by Honduras. Equally important it is to understand the efforts of the government of the Republic to manage its resources and institutions so that the cultural rights are fully guaranteed.

Palabras Clave: Cultura, Acceso a la Cultura, Derechos Constitucionales, Derechos Humanos.

KEYWORDS: Culture, Access to culture, Constitutional Rights, Human Rights.

\footnotetext{
* Trabajo recibido el 30 de mayo de 2016 y aprobado del 17 de mayo de 2017.

** Abogada, Máster en Derecho Empresarial de la Universidad Tecnológica Centroamericana (Honduras), Máster en Derecho, Empresa y Justicia de la Universidad de Valencia (España), Doctoranda en Estudios Jurídicos, Ciencia Política y Criminología de la Universidad de Valencia (España). Profesora de la Carrera de Relaciones Internacionales.
} 


\section{INTRODUCCIÓN}

El presente estudio es un análisis jurídico-descriptivo, que permite entender cómo es que el ordenamiento jurídico hondureño configura el derecho a la cultura, para determinar posteriormente por medio de un análisis correlacional, si el Estado de Honduras garantiza efectivamente dicho derecho.

Este documento descansa en una búsqueda y revisión documental exhaustiva de los instrumentos jurídicos vigentes en Honduras, sirviendo como marco de referencia del estudio. Vale señalar que estos instrumentos proceden del Derecho interno del Estado de Honduras, así como del Derecho Internacional. Posteriormente, el análisis se enfocará en la revisión exhaustiva, también, de la documentación disponible en los portales gubernamentales de las instituciones de la administración pública, incluyendo en éstos los portales de transparencia, para poder deducir cuáles son los mecanismos de gestión cultural utilizados por el actual gobierno.

La hipótesis propuesta para el estudio establece que las medidas adoptadas por el Estado de Honduras con relación a la protección, respeto y promoción de la cultura no son suficientes para lograr la plena efectividad del derecho a la cultura de los ciudadanos. De ahí que la metodología utilizada incluya una revisión del texto constitucional y de los tratados internacionales ratificados por Honduras en la materia, así como de los instrumentos de planificación del presente gobierno para determinar cuáles son las acciones que la presente administración está ejecutando.

\section{El Derecho a la Cultura en la Constitución hondureña}

Ruiz-Rico Ruiz señala que el concepto de cultura, como objeto de estudio del Derecho constitucional, es indeterminado a la vez que es inexcusable, al constituirse como un verdadero reto para los constitucionalistas por su carácter polisémico y elástico ${ }^{1}$. La complejidad detrás de dicho concepto deviene de situaciones tales como el período histórico en el que se vive, el sentimiento general de la población, el momento y modo en el que es asumido por el Estado, pero sobre todo, por la función que le es asignada en un determinado contexto socio-político, y por el valor que se le atribuye ${ }^{2}$.

1 Ruiz-Rico Ruiz (2005), p. 10.

2 Dominici (2005), p. 159. 
La Constitución ${ }^{3}$ reconoce la importancia que debe tener la cultura en la vida ciudadana de los hondureños, quedando patentizada ampliamente en dicho texto. Desde su inicio, en el artículo 1, la Carta Magna señala que una de las razones que justifican la existencia misma del Estado de Honduras es la de asegurar a sus habitantes el goce de ésta. Es decir, el Estado, para justificarse a sí mismo, debe garantizar a los ciudadanos el derecho al acceso a la cultura. Que el constituyente haya decidido incluir desde ese primer artículo el tema cultural, puede ser un indicativo de la autonomía que éste quiso imprimirle a la temática ${ }^{4}$.

Sin embargo, si se continúa revisando el texto constitucional, es evidente que el legislador obvió garantizar taxativamente ese derecho, mucho menos explicar su alcance. No obstante, eso no quiere decir que éste no la tutele. Lo que sucede es que la protección de las libertades culturales, tradicionalmente, se ha entendido circunscrita a varios de los derechos que se encuentran reconocidos en la Constitución, tales como la libertad de enseñanza, la de prensa y la de expresión 5 .

No obstante, la referencia a la cultura antes señalada en el artículo 1 no es la única que aparece en el texto constitucional. En el artículo 75 se encuentra el reconocimiento de la existencia de valores éticos y culturales. El artículo 151, por su parte, establece que una función prioritaria del Estado, como ser la educación, tiene como único propósito declarado, conservar, fomentar y difundir la cultura. Además, constitucionalmente también se vincula a parte de la institucionalidad del país con la cultura, tal como sucede con el artículo 160, cuando le asigna a la Universidad Nacional Autónoma de Honduras la función de contribuir a su difusión general.

El Estado también se ha autoimpuesto obligaciones vinculadas con la cultura. Estas obligaciones son:

a. Infraestructurales: Creación de centros culturales (art. 170).

b. Regulatorias: Normas para la conservación, restauración, mantenimiento y restitución del patrimonio cultural (art. 172).

c. Difusión y Promoción: Difusión de la cultural como instrumento de educación extraescolar, de la cultura física y los deportes (art. 174), de producciones de autores nacionales o extranjeros que contribuyan al desarrollo nacional (art. 175).

d. Preservación y estimulación: De las culturas nativas (art. 173).

3 República de Honduras (1982), p. 1.

4 González Moreno (2003), pp. 138-144.

5 Ruiz-Rico Ruiz (2005), p. 10. 
También impone la Constitución obligaciones que alcanzan a terceros, como en el caso de los patronos, cuando establece que una de las garantías que tienen los trabajadores en el país es que su salario sea suficiente para cubrir las necesidades materiales y culturales de su hogar (art. 128); o el caso de los medios de comunicación privados, los que deben coadyuvar con la educación y la cultura (art. 176) ${ }^{6}$.

$\mathrm{Al}$ interpretar la norma constitucional es posible deducir su finalidad, y de esta finalidad, recabar un criterio de decisión 7 . De ahí que, a pesar que el derecho a la cultura no esté incluido taxativamente en la Constitución, es posible extraer su ratio iuris, y afirmar que dicho derecho sí está garantizado, aunque sea de manera indirecta. Sin embargo, para identificar que dichos intereses han sido relevantes para el constituyente es necesario contar con elementos suficientes que puedan ser conjugados, como se ha podido establecer que sucede con relación al derecho a la cultura en el análisis antes realizado. Lo mismo sucede con otros derechos, como, por ejemplo, el derecho a un ambiente sano y equilibrado que tampoco está explícitamente garantizado en la Constitución, sino que se puede interpretar éste indirectamente, por medio del derecho a la salud.

En otras latitudes se han utilizado otros métodos interpretativos, vinculando jurisprudencialmente el derecho a la cultura con el derecho a la libre expresión. Este es el caso español, donde el Tribunal Constitucional ha dicho en Sentencia del 16 de marzo de 1981 que los poderes públicos no pueden injerir en el campo de la creación artística, y además deben remover cualquier obstáculo que pueda limitar dicha creación ${ }^{8}$. En el caso de Honduras, el derecho a la libre expresión o a la libre emisión del pensamiento está contenido en el artículo 72 del texto constitucional, pero no se ha encontrado jurisprudencia hondureña que haga esta misma relación?

La interpretación propuesta deja ver que, de manera tal vez tímida, el constituyente hondureño reconoce y garantiza el derecho a la cultura. Esa timidez no deja de ser cuestionable pues este es un derecho esencial para cualquier individuo, pues determina su pertenencia a la colectividad donde ha de desenvolverse política, jurídica y económicamente ${ }^{10}$. A la vez que la integración hecha a partir de varias normas constitucionales, si bien sirve para reconocer la existencia del derecho,

6 República de Honduras (1982), pp. 5-10.

7 Picontó Novales (1992), p. 229.

8 Sala Segunda del Tribunal Supremo Constitucional, Sentencia 6/1981, de 16 de marzo de 1981.

9 República de Honduras (1982), p. 5.

10 Núñez Rivero y Pérez A. (2015), p. 60. 
no es suficiente para discernir su amplitud. Para eso es necesario poder deducir el contenido del concepto "cultura".

Desde una perspectiva académica, la cultura puede definirse como el conjunto de componentes que conforman a una sociedad, incluyendo creencias, convicciones, idiomas, etc. ${ }^{11}$; sin embargo, el texto constitucional hondureño no ofrece un concepto definitivo desde el ámbito jurídico. Cuando mucho, llega a explicar qué es lo que está contenido en la noción de patrimonio cultural de la nación, indicando en el artículo 172 que "toda riqueza antropológica, arqueológica, histórica y artística de Honduras" forma parte de éste ${ }^{12}$. Vale hacer notar que este artículo deja entrever que para el constituyente hondureño, el concepto de patrimonio cultural tiene un contenido puramente material ${ }^{13}$.

No obstante, es necesario aclarar que carecer de ese concepto no imposibilita el reconocimiento previo del derecho ${ }^{14}$, ni contradice la interpretación hecha por medio de la integración normativa. De hecho, hay corrientes de la antropología que se manifiestan en contra de delimitarlo como un concepto fijo ${ }^{15}$. Sin embargo, también es válido decir que el concepto de cultura se va conformando en la medida que se va instrumentalizando, y esa es una tarea que le corresponde a los poderes políticos de una sociedad ${ }^{16}$.

La Constitución tampoco establece una sistematización jerárquica de los derechos garantizados a la ciudadanía, que explique cuál es el juego del derecho a la cultura con relación a los demás derechos contenidos en el catálogo constitucional. Es por eso que, para completar el análisis anterior, es obligatorio referirse a los constructos relacionados con la materia que han sido desarrollados por el Derecho Internacional Público.

\section{El Derecho a la Cultura en los Tratados Internacionales RATIFICADOS POR HONDURAS Y DEMÁS INSTRUMENTOS INTERNACIONALES}

Abocarse el Derecho Internacional Público es -sin duda- necesario en un trabajo de esta naturaleza, porque los tratados internacionales son fuentes reconocidas del

11 Núnez Rivero y Pérez A. (2015), p. 58.

12 República de Honduras (1982), p. 10.

13 García Fernández (2005), p. 46.

14 Dorantes Díaz (2013), p. 846

15 CoWAn (2010), pp. 68-69.

16 Donders (2010), p. 15. 
Derecho. En el caso que nos ocupa, su importancia radica en que ser los instrumentos regulatorios que crean derechos y obligaciones para los Estados que los suscriben ${ }^{17}$. Considerando lo anterior, el análisis de estas fuentes debe partir por establecer cuál es el mecanismo de recepción de tratados internacionales disponible en Honduras. En ese sentido, la Constitución hondureña contiene en el artículo 18 un sistema de recepción de los tratados internacionales, incorporándolos al Derecho interno y haciéndolos prevalecer por sobre la ley ${ }^{18}$. Además, la legislación se somete al control de convencionalidad, permitiendo a los jueces utilizar al derecho internacional como herramienta de contraste para el derecho interno ${ }^{19}$.

Ante la falta de claridad del constituyente frente al reconocimiento y amplitud del derecho a la cultura, el control de constitucionalidad se vuelve la herramienta idónea. Éste ha quedado establecido en el artículo 2 del Pacto Internacional de Derecho Civiles y Políticos, suscrito por Honduras el 19 de diciembre de 1966 y ratificado el 25 de agosto de 1997; en el artículo 2 del Pacto Internacional de los Derechos Económicos, Sociales y Culturales, suscrito por Honduras el 19 de diciembre de 1966 y ratificado el 17 de febrero de 1981; y en los artículos 1 y 2 de la Convención Americana de los Derechos Humanos, la que Honduras suscribió el 22 de noviembre de 1969 y lo ratificó el 8 de septiembre de 1977.

El control de convencionalidad ha sido reconocido por la jurisprudencia de la Corte Suprema de Justica, siendo el ejemplo más reciente la Sentencia RI1343-14 de 22 de abril de 2015. En dicha sentencia la Corte ha recalcado que si bien no tiene la atribución de reformar la Constitución, sí es función suya la interpretación de dicha norma, siendo una de las herramientas disponibles para dicho menester el bloque de convencionalidad. También ha señalado que dicho bloque es particularmente útil cuando la norma originaria colisiona con los principios y normas internacionales de Derechos Humanos.

En estos supuestos, ha dicho la Corte Suprema, las normas constitucionales impuganadas pierden su aplicabilidad al ser incompatibles con dichos tratados, convenciones y pactos internacionales, pues la Constitución debe ser interpretada en el marco de dicho bloque de convencionalidad, tomando en consideración el principio pro homine, así como el artículo 59 de la Constitución hondureña, que establece que la persona humana es el fin supremo de la sociedad y del Estado ${ }^{20}$.

17 García Fernández (2005), p. 67.

18 República de Honduras (1982), p. 2.

19 Carbonell (2013), p. 71.

20 Sala de lo Constitucional, Sentencia RI-1343-14, de 22 de abril de 2015. 
Lo anterior es además obvio si las normas internacionales en materia de derechos humanos habían sido ratificadas por Honduras antes de la entrada en vigencia de la actual Constitución. Este es precisamente el caso del ejemplo siguiente. La primera obligación internacional vinculada al derecho a la cultura del Estado de Honduras se encuentra en el Pacto Internacional de Derechos Económicos, Sociales y Culturales. El artículo 15 de dicho instrumento señala que "1. Los Estados partes en el presente Pacto reconocen el derecho de toda persona a: a) Participar en la vida cultural; b) Gozar de los beneficios del progreso científico y de sus aplicaciones; c) Beneficiarse de la protección de los intereses morales y materiales que le correspondan por razón de las producciones científicas, literarias o artísticas de que sea autora..."21. La ratificación por parte de Honduras del Pacto Internacional antes mencionado el 17 de febrero de 1981 es el que garantiza, ahora sí, de manera directa, el derecho al acceso a la cultura. La Constitución hondureña está vigente desde 1982.

Vale decir que no es el Pacto el primer instrumento internacional donde se reconoce este derecho. Es necesario hacer referencia a la Declaración Universal de los Derechos Humanos, la que en su artículo 27 reconoce que toda persona tiene derecho a tomar parte, gozar y participar de la cultura, así como a que se le protejan los derechos morales y materiales que le corresponden en función de su producción cultural ${ }^{22}$. Lo anterior deja claramente establecido que el derecho a la cultura es considerado fundamental ${ }^{23}$. Además, vincula al derecho a la cultura con el concepto universal de la dignidad humana como base normativa de los derechos de índole social ${ }^{24}$. Sin embargo, esta es una declaración de derecho blando, que no genera verdaderas obligaciones para los Estados, sino que son estándares que deberán ser alcanzados por los miembros de la organización ${ }^{25}$.

En el ámbito regional también hay instrumentos internacionales que regulan el derecho a la cultura. La Carta de la Organización de los Estados Americanos, ratificada por Honduras el 13 de enero de 1950, señala en el artículo 30 que la cultura es un elemento del desarrollo integral de los pueblos. Entre los diversos compromisos estatales adquiridos por medio de este instrumento, se incluye la

\footnotetext{
21 Organización de las Naciones Unidas (1966), p. 5.

22 Organización de las Naciones Unidas (1948).

23 Núnez Rivero y Pérez A. (2015), p. 59.

24 Dorantes díaz (2013), p. 850.

25 DONDERS (2010). p. 15.
} 
incorporación y creciente participación de los sectores marginales de la población, en los diferentes ámbitos de la vida ciudadana, incluyendo el cultural, como mecanismo de consolidación del régimen democrático. Otros de esos compromisos son el de darle primordial importancia dentro de sus planes de desarrollo al estímulo de la cultura y asegurar los bienes de la cultura a la totalidad de la población. Además, se reconoce la importancia de la contribución de las asociaciones culturales a la vida de la sociedad y al proceso de desarrollo ${ }^{26}$.

Por su parte, el Pacto de San José, o Convención Americana sobre Derechos Humanos, establece en su artículo 26 que "los Estados Partes se comprometen a adoptar providencias, tanto a nivel interno como mediante la cooperación internacional, especialmente económica y técnica, para lograr progresivamente la plena efectividad de los derechos que se derivan de las normas económicas, sociales y sobre educación, ciencia y cultura, contenidas en la Carta de la Organización de los Estados Americanos, reformada por el Protocolo de Buenos Aires, en la medida de los recursos disponibles, por vía legislativa u otros medios apropiados" 27 . Honduras lo suscribió el 22 de noviembre de 1969 y lo ratificó el 8 de septiembre de 1977.

Con lo anterior, y en virtud de la recepción que el texto constitucional hace de los tratados internacionales y de la aplicabilidad del control de convencionalidad, es posible afirmar contundentemente que la ciudadanía hondureña tiene garantizado el derecho al acceso a la cultura. Sin embargo, tampoco los instrumentos arriba citados incluyen una definición contundente para el término cultura, aunque sí permiten ir entendiendo algunas de sus características, tales como que este concepto engloba a las artes, la literatura, los progresos intelectuales y los descubrimientos científicos, y está conformada por bienes de diversa índole.

Vale señalar que además de los instrumentos arriba relacionados, existe otro grupo de tratados internacionales suscritos y ratificados por Honduras que regulan el derecho a la cultura, y que contienen otra serie de obligaciones internacionales adquiridas por este Estado. Por ejemplo, en 1954, se suscribe la Convención de La Haya para la protección de la propiedad cultural en caso de conflicto armado ${ }^{28}$. Honduras se adhiere a ella el 25 de octubre de 2002, 48 años después. En 1970, se suscribe la Convención de París sobre las medidas que deben adoptarse para

26 Organización de los Estados Americanos (1948), pp. 9-14.

27 Organización de los Estados Americanos (1969), p. 10.

28 Organización de las Naciones Unidas para la Educación, la Ciencia y la Cultura (1954). 
prohibir la importación, la exportación y la transferencia de propiedades ilícitas de bienes culturales ${ }^{29}$. Honduras la ratifica el 19 de marzo de 1979, 9 años después. En 1972, también en París, se suscribe la Convención sobre la Protección del Patrimonio Mundial Cultural y Natural ${ }^{30}$. Honduras la ratifica siete años después, el 8 de junio de 1979. En el año 2001, se suscribe la Convención sobre la Protección del Patrimonio Cultural Subacuático ${ }^{31}$, ratificada por Honduras el 23 de julio de 2010, 9 años después.

En 2003 se suscribe en París, la Convención para la Salvaguardia del Patrimonio Cultural Inmaterial. Fue ratificada por Honduras el 24 de julio de 2006, 3 años después. La Convención enumera como manifestaciones del patrimonio cultural inmaterial las tradiciones y expresiones orales, incluido el idioma como vehículo del patrimonio cultural inmaterial; las artes del espectáculo; los usos sociales, rituales y actos festivos; los conocimientos y usos relacionados con la naturaleza y el universo, y las técnicas artesanales tradicionales ${ }^{32}$. En París también, en el 2005, se suscribió la Convención sobre la protección y promoción de la diversidad de las expresiones culturales ${ }^{33}$. Honduras se adhirió el 31 de agosto de 2010, 5 años después.

Si bien es cierto la ratificación de estos tratados es posterior a 1982, el artículo 15 constitucional claramente establece que Honduras hace suyos los principios y prácticas del Derecho Internacional, de ahí que es ineludible el control de convencionalidad antes expuesto ${ }^{34}$. Además, es de señalar la dilación con la que el Estado de Honduras ha ratificado estos instrumentos, y aunque van dando forma al contenido de la categoría "cultura" no ofrecen un concepto definitivo, denotando la naturaleza difusa del derecho ${ }^{35}$. Lo que sí, es que hacen aportes significativos para construir el concepto del patrimonio cultural y el concepto de bienes culturales, en los que se ahondará posteriormente.

Más allá de los instrumentos de cumplimiento obligatorio ya analizados, también es necesario referirse a las resoluciones de los organismos internacionales.

29 Organización de las Naciones Unidas para la Educación, la Ciencia y la Cultura (1970).

30 Organización de las Naciones Unidas para la Educación, la Ciencia y la Cultura (1972).

31 Organización de las Naciones Unidas para la Educación, la Ciencia y la Cultura (2001a).

32 Organización de las Naciones Unidas para la Educación, La Ciencia y la Cultura (2003a), pp. 2-3.

33 Organización de las Naciones Unidas para la Educación, la Ciencia y la Cultura (2005).

34 República de Honduras (1982), p. 2.

35 Dorantes Díaz (2013), pp. 853-855. 
Por ejemplo, uno de los vacíos constitucionales antes planteado con relación al derecho a la cultura, es aclarado por la resolución 32/130 de 16 de diciembre de 1977 de la Asamblea General de la Organización de las Naciones Unidas, donde establece el juego que el derecho a la cultura tiene como los demás derechos humanos al señalar que "a) todos los derechos humanos y libertades fundamentales son indivisibles e interdependientes; debe prestarse igual atención y consideración urgente a la implantación, promoción y protección de todos los derechos humanos, tanto civiles y políticos, como económicos, sociales y culturales; b) la plena consecución de los derechos civiles y políticos es imposible sin el disfrute de los derechos económicos, sociales y culturales..."36.

Si bien es cierto la resolución de un organismo internacional no califica como tratado internacional, obtienen su autoridad normativa de los tratados instituyentes de dichas organizaciones. En el caso de las resoluciones de la Asamblea General de Naciones Unidas no son obligatorias por sí mismas para los Estados miembros, en el caso de resoluciones como la antes citada, donde se dota de contenido a categorías creadas por el Derecho Internacional se reconoce como opinio iuris colectiva ${ }^{37}$.

También existen instrumentos de soft law que no son jurídicamente vinculantes, pero que dotan de definiciones contundentes del término cultura, siendo necesario referirse a ellos ${ }^{38}$. Un ejemplo de esto es la Declaración de la Unesco sobre Diversidad Cultural de 2 de noviembre de 2001. Esta declaración la define como “... el conjunto de rasgos distintivos espirituales y materiales, intelectuales y afectivos que caracterizan a una sociedad o a un grupo social y que abarca, además de las artes y las letras, los modos de vida, manera de vivir juntos, los sistemas de valores, las tradiciones y las creencias”. Señala además que “... adquiere formas diversas a través del tiempo y del espacio" 39.

Esto se puede complementar con lo establecido en la Recomendación relativa a la participación y la contribución de las masas populares en la vida cultural, 26 de noviembre de 1976, que señala entre otros aspectos que la cultura forma parte integrante de la vida social, y que es, por su propia naturaleza, un fenómeno social, el resultado de la creación común de los hombres y de la acción que ejercen

36 Organización de las Naciones Unidas (1977), pp. 160-161.

37 Pastor Ridruejo (2015), pp. 156-160.

38 Mazuelos Bellido (2004), p. 2.

39 Organización de las Naciones Unidas para la Educación, la Ciencia y la Cultura (2001b). 
unos sobre otros. También señala que la cultura se considera cada vez más como un componente importante de la vida humana y uno de los principales factores del progreso, dejando de ser únicamente una acumulación de obras y de conocimientos que produce, compila y conserva una minoría selecta para ponerlos al alcance de todos. Establece que ésta no se limita al acceso a las obras de arte y a las humanidades sino que es a la vez adquisición de conocimientos, exigencia de un modo de vida, necesidad de comunicación, ampliando el concepto a todas las formas de creatividad y de expresión de los grupos o los individuos, ya sea en sus modos de vida o en sus actividades artísticas ${ }^{40}$.

La amplia gama de instrumentos internacionales culturales permite darles contenido a otros conceptos también relevantes para dimensionar integralmente el concepto de "cultura", comenzando por el de "bienes culturales". Este es un concepto amplísimo que incluye a muebles e inmuebles que sean significativos para el patrimonio cultural de los pueblos ${ }^{41}$. Dicha significancia es determinada por el valor artístico, histórico o científico que estos posean, y que muchas veces va a ser determinado a partir de la precisión con la que se conozca su origen e historia. Pero además su valor no es intrínseco, sino que incluye el marco circundante de dichos bienes ${ }^{42}$. Es precisamente ese valor el que hace que queden fuera del comercio de los hombres y no puedan ser asumidos como bienes ordinarios de consumo, pues constituyen así el elemento fundamental de la personalidad de los pueblos ${ }^{43}$.

La noción de patrimonio cultural también es amplia. Este es entendido como un componente importante de la identidad cultural de los comunidades, los grupos y los individuos, y de la cohesión social ${ }^{44}$, conformado por bienes culturales ${ }^{45}$, incluyendo los que están bajo el agua ${ }^{46}$, así como por manifestaciones culturales inmateriales ${ }^{47}$. Estos bienes culturales deberán ser gestionados por instituciones culturales reconocidas por la autoridad pública competente ${ }^{48}$. Estas instituciones

40 Organización de las Naciones Unidas para la Educación, la Ciencia y la Cultura (1976a).

41 Organización de las Naciones Unidas para la Educación, la Ciencia y la Cultura (1954).

42 Organización de las Naciones Unidas para la Educación, la Ciencia y la Cultura (1970).

43 Organización de las Naciones Unidas para la Educación, la Ciencia y la Cultura (2001b).

44 Organización de las Naciones Unidas para la Educación, la Ciencia y la Cultura (2003b).

45 Organización de las Naciones Unidas para la Educación, la Ciencia y la Cultura (1954).

46 Organización de las Naciones Unidas para la Educación, la Ciencia y la Cultura (2001a).

47 Organización de las Naciones Unidas para la Educación, la Ciencia y la Cultura (2003a), pp. 2-3.

48 Organización de las Naciones Unidas para la Educación, la Ciencia y la Cultura (1976b). 
son establecimientos permanentes administrados en función del interés general, con miras a conservar, estudiar, valorizar y poner al alcance del público dichos bienes culturales.

Dicha gestión deberá realizarse en el marco de políticas culturales que garanticen la libre circulación de las ideas y las obras, y creen condiciones propicias para la producción y difusión de bienes y servicios culturales diversificados ${ }^{49}$. Esto implica el fomento de industrias culturales que dispongan de medios para desarrollarse en los planos local y mundial. Pero, además, debe incluir formas de protección material y jurídica que sólo pueden ser obtenidas, la primera, por medio de la tecnología, y las segundas, por medio de la ley ${ }^{50}$.

Estas políticas y este modelo de gestión debe garantizar la diversidad cultural, que es entendida como una de las fuentes de desarrollo, no solamente económico, sino que también individual, como medio de acceso a una existencia intelectual, afectiva, moral y espiritual satisfactoria, integrándolo por lo tanto como un elemento importante del concepto del desarrollo sostenible, entendiendo como tal, el modelo de desarrollo que logra satisfacer las necesidades presentes, sin comprometer la satisfacción de las necesidades de las generaciones futuras ${ }^{51}$. Dicha diversidad cultural es necesaria e inseparable del respeto de la dignidad de la persona humana, volviéndose, por lo tanto, su defensa, un imperativo ético. $\mathrm{Al}$ trascender del plano puramente económico, es entendible que las fuerzas del mercado por sí solas no pueden garantizar la preservación y promoción de dicha diversidad, reto que debe ser abordado por las políticas públicas estatales, las cuales deben integrar en sus procesos de gestión tanto al sector privado, como a la sociedad civil ${ }^{52}$.

De estos instrumentos internacionales también se desprende el concepto de "acceso a la cultura", entendido como "la posibilidad efectiva para todos, principalmente por medio de la creación de condiciones socioeconómicas, de informarse, formarse, conocer, comprender libremente y disfrutar de los valores y bienes culturales" 53 . Para que el acceso a los bienes culturales antes definidos esté al alcance de amplias capas de la población, deben reunirse las condiciones

49 Organización de las Naciones Unidas para la Educación, la Ciencia y la Cultura (2001b).

50 García Fernández (2005), p. 48.

51 Comisión Mundial de Ambiente y Desarrollo (1987), p. 16.

52 Organización de las Naciones Unidas para la Educación, la Ciencia y la Cultura (2001b).

53 Organización de las Naciones Unidas para la Educación, la Ciencia y la Cultura (1976a). 
económicas que permitan a los interesados no sólo disfrutar de esos bienes, sino también participar activamente en todas las manifestaciones de la vida cultural, así como en el proceso del desarrollo en este ámbito. La eliminación de las desigualdades económicas y sociales, que impiden a amplias capas de la población acceder a los conocimientos científicos que son el fundamento de la ciencia y la tecnología y tener conciencia de sus propias necesidades culturales, es condición para ampliar el acceso a la vida cultural y la participación en ella ${ }^{54}$.

De hecho, el acceso a la cultura y la participación en la vida cultural son dos aspectos complementarios de una misma realidad percibida en la reciprocidad de sus efectos, ya que el acceso puede favorecer la participación en la vida cultural, y la participación puede ensanchar el acceso a la cultura al darle su verdadero sentido. En otras palabras, sin participación, el mero acceso a la cultura está necesariamente muy por debajo de los objetivos del desarrollo cultural. Para aclarar lo anterior, vale establecer que por participación en la vida cultural se entiende "la posibilidad efectiva y garantizada para todo grupo o individuo de expresarse, comunicar, actuar y crear libremente, con objeto de asegurar su propio desarrollo, una vida armoniosa y el progreso cultural de la sociedad" 55 .

El acceso y la participación, que deben dar a todos y cada uno la posibilidad no solamente de recibir sino también de expresarse en todos los campos de la vida social, suponen la máxima libertad y tolerancia en la formación, la creación y la difusión culturales. El objetivo último del acceso y la participación es elevar al nivel espiritual y cultural de toda la sociedad sobre la base de los valores humanos, y dar a la cultura un contenido democrático humanitario ${ }^{56}$. La participación en la vida cultural presupone la afirmación del individuo, de su dignidad y su valor, así como el ejercicio de las libertades y los derechos fundamentales del hombre. Se traduce en una afirmación de identidad, de autenticidad y de dignidad ${ }^{57}$.

Esta forma de participación en la vida cultural requiere de los Estados que diseñen políticas públicas en los ámbitos del desarrollo, de la educación permanente, de la ciencia y la tecnología, de progreso social, del ambiente, de la comunicación, y de la cooperación internacional, de tal forma que se asegure el crecimiento económico y la justicia, que asegure su educación cultural y su formación artística, que

54 Organización de las Naciones Unidas para la Educación, la Ciencia y la Cultura (1976a).

55 Organización de las Naciones Unidas para la Educación, la Ciencia y la Cultura (1976a).

56 Organización de las Naciones Unidas para la Educación, la Ciencia y la Cultura (1976a).

57 Organización de las Naciones Unidas para la Educación, la Ciencia y la Cultura (1976a). 
proteja la identidad cultural de los pueblos, que trate de reducir las desigualdades que afectan a ciertos grupos y personas, que cree un marco de vida propicio a la plena expansión de los individuos y de las comunidades, que fortalezca el libre intercambio de informaciones, de ideas y de conocimientos, y que esté basada en los principios de la igualdad de las culturas, el respeto, la comprensión y la confianza mutuos y el fortalecimiento de la $\mathrm{paz}^{58}$.

\section{La Gestión Cultural en Honduras}

Un Estado que reconoce y garantiza los derechos culturales para su ciudadanía debe poder lograr un equilibrio entre dos elementos que son de distinta naturaleza: la libertad de las personas a manifestarse culturalmente, y el control que debe ser ejercido por los poderes públicos. Para esto, será necesario entender el rol que cumplen los poderes públicos en este contexto, siendo éste eminentemente político. Esto significará que el Estado, de alguna forma u otra, tendrá que entremeterse en la esfera cultural ${ }^{59}$. A continuación se discute dos formas de intervención: la planificación de la actividad cultural, y su financiamiento por parte del Estado.

\section{A. La Planificación de la Gestión Cultural}

Para entender los avances obtenidos por el Estado de Honduras en materia de acceso a la cultura, es necesario referirse al sistema de planificación nacional. Dicho sistema tiene desde el 2012 una hoja de ruta clara, denominado "Plan de Nación, Visión de País”. Dentro de los principios que informan a este instrumento de planificación se incluye el "Respeto y preservación de la cultura y costumbres de los grupos étnicos”. Dicho principio supone el reconocimiento de Honduras como un Estado multicultural y plurilingüe, siendo uno de sus compromisos impulsar y fomentar su desarrollo. Pero no se hace ninguna referencia a la cultura tradicional o popular ${ }^{60}$.

En el Plan de Nación, la educación y la cultura son vistas estratégicamente como medios de emancipación social. Para esto, se propone la descentralización de la cultura, entendida como "el conjunto de instancias y procesos de desarrollo institucional y comunitario que, a través de mecanismos de planificación, ejecu-

58 Organización de las Naciones Unidas para la Educación, la Ciencia y la Cultura (1976a).

59 González Moreno (2003), pp. 61-62.

60 República de Honduras (2010), p. 20. 
ción, y seguimiento articulados entre sí, facilitan el desarrollo cultural y el acceso a la comunidad de los bienes y servicios culturales, según los principios de descentralización, participación, interculturalidad, autonomía, equidad y concertación”. Sin embargo, transfiere esa responsabilidad de la construcción de una democracia basada en la interculturalidad a "la gente", y debe tener como propósito "promover y lograr la consolidación de una cultura de la participación, descentralización, concertación y transparencia donde se valore el consenso social por encima de los intereses particulares y privados". Ese proceso de descentralización debe ser flexible, de tal forma que sea participativo e igualitario. Para eso se plantea una serie de desafíos es materia de cultura para la construcción de identidad ${ }^{61}$.

Estos desafíos incluyen la integración de consejos regionales de cultura, adscritos a los consejos regionales de desarrollo. Dichos espacios deberán servir para la toma de decisión sobre programas y presupuestos, siendo su principal función la creación de estrategias regionales de cultura, elaboración de propuestas y proyectos y gestión de recursos para actividades culturales. En ellos se concertará la política nacional de cultura ${ }^{62}$.

También se incluye la promoción de expresiones artísticas y culturales. Para ello, se mejorarán los centros académicos para la formación de artistas en todas las regiones del país, en la inversión pública se incluirá componentes para la promoción y apoyo de los artistas hondureños y el Estado tutelará y patrocinará las organizaciones culturales. Igualmente, la promoción de grupos artísticos y apoyo a las iniciativas empresariales de apoyo a las artes y construcción de instalaciones para ampliar el acceso de los ciudadanos a las manifestaciones culturales. Para ello, las políticas de Estado procurarán favorecer iniciativas de responsabilidad social empresarial para que el sector privado colabore con las iniciativas ${ }^{63}$.

Para el año 2022, se avizora la consolidación de los consejos regionales de cultura, el crecimiento de la producción artística en el país, el 25\% de los grupos étnicos habrán participado en proyectos de desarrollo cultural y se habrá aumentado el acceso de la población a centros de difusión cultural. Para 2034, la producción artística hondureña será difundida internacionalmente y el país se habrá consolidado en la región como generador de obras artísticas y literarias de excelencia. Se habrá establecido un sistema de concursos y galardones a nivel regional. Para

61 República de Honduras (2010), pp. 46-70.

62 República de Honduras (2010), p. 71.

63 República de Honduras (2010), pp. 71-72. 
el año 2038 se prevé un país con "un medio cultural artístico y vibrante" que se habrá incorporado a la cultura e identidad nacional ${ }^{64}$.

Sin embargo, en el informe preliminar de avances publicado por la Unidad de Apoyo Técnico Presidencial en octubre de 2013, sólo se reportó que en el 2012, mediante el Programa Nacional de Educación para las Etnias Autóctonas y Afroantillanas de Honduras (Proneeaah), se ha implementado el Modelo de Educación Intercultural Bilingüe en 161 Centros Educativos de Pre-Básica y Básica, asimismo, se certificó 300 niños en dicho modelo educativo ${ }^{65}$.

Por medio del Decreto Ejecutivo PCM 078-2014 se oficializa el Plan "Todos por una Vida Mejor" como instrumento de planificación para el período presidencial 2014-2018. En él se declara que uno de los cuatro propósitos en los que el plan se fundamenta es en el desarrollo humano, reducción de desigualdad y protección social ${ }^{66}$. Vinculado a éste, en abril de 2014 se publica el "Plan estratégico del Gobierno, 2014-2018", disponible en los portales de transparencia. En dicho plan, la única mención que se hace de la cultura, está vinculado con las actividades de promoción que le corresponden al Servicio Exterior ${ }^{67}$.

A lo anterior se puede agregar que, por medio del Decreto No 266-2013 desaparece la Secretaría de Cultura, Artes y Deportes, al integrarse a la ahora denominada Secretaría de la Presidencia ${ }^{68}$. Dicha integración supuso la degradación de la Secretaría a una mera Dirección Ejecutiva. En la memoria de labores de la Secretaría para el 2014, en la única referencia que hace a las actividades culturales, reporta que, como parte de un trabajo especial, se realizaron evaluaciones psicométricas documentales y desempeño de todo el personal de la antigua Secretaría de Cultura, Artes y Deportes ${ }^{69}$.

En el Plan Operativo Anual de 2015 de la Secretaría de la Presidencia se reportan los siguientes proyectos:

1. Proyecto Corredor del Quetzal.

2. Proyecto de Nutrición y Protección Social.

64 República de Honduras (2010), pp. 72-73.

65 Secretaría de Estado en el Despacho Presidencial (2013), p. 68.

66 República de Honduras (2014), p. 15.

67 Presidencia de la República (2014), p. 39.

68 República de Honduras (2013), p. 23.

69 Secretaría de Estado en el Despacho Presidencial (2014), p. 22. 
3. Proyecto para el Desarrollo de Microempresas para Pobres.

4. Programa de Apoyo a la Presidencia de la República.

5. Proyecto de Protección Social en Apoyo al Programa Bono Diez Mil-PRAF.

6. Programa de educación primaria e integración tecnológica.

7. Proyecto de dotación de equipo médico para la sala de cardiología y pediatría.

8. Proyecto Unidad Móvil de Transmisión de Televisión Educativa Nacional

9. Proyecto Construyendo Sonrisas.

10. Proyecto de Fortalecimiento de la Secretaría del Despacho Presidencial ${ }^{70}$.

Ninguno de ellos directamente vinculados con el acceso a la cultura o la participación de los hondureños en la vida cultural.

\section{B. El Financiamiento de la Gestión Cultural}

En el Presupuesto General de Egresos de la Administración Central para el ejercicio 2015, se asignó L. 22.519.441.503 para “Educación, investigación, cultura y actividades recreativas". De las instituciones de la Administración Central a las que estaban asignados esos fondos, la única que está vinculada a la gestión cultural es el Centro de la Cultura Garinagu, a la que se destinaron L. 7.788.692, es decir, el $0.03 \%{ }^{71}$.

Al revisar el Presupuesto de Egresos por Finalidad y Función de la Administración Central se observa una disminución en las asignaciones para actividades culturales, pues en el año 2013 se había aprobado el monto de L. 18.674.296 para este renglón, para el año 2014 se aprobó L. 18.212.433, disminuyéndose en un $2.5 \%$, y para el año 2015 no se aprobó cantidad alguna. Igual suerte sufre el renglón denominado "Educación, investigación, cultura y actividades recreativas”, que para el año 2013 tenía asignados L. 5.402.222.050, para el año 2014 se asignó L. 4.889.939.037 disminuyendo en 9.5\%, y para el año 2015, L. 4.251.000.037, disminuyendo en un $21.45 \%$ con relación al 2013, y un $13.1 \%$ con relación al $2014^{72}$.

Sin embargo, en el Presupuesto de Egresos por Programa, se le asigna al renglón de "Cultura, Arte y Deportes" de la Secretaría de la Presidencia L. 156.201.426, que significa un aumento en relación al presupuesto asignado en

70 Secretaría de la Presidencia (2015).

71 Secretaría de Finanzas (2015b), p. 2.

72 Secretaría de Finanzas (2015a), p. 1. 
los años 2013 y 2014 a la extinta Secretaría de Cultura, Artes y Deportes, que ascendió a L. 153.290 .430 y L. 104.944.159, respectivamente ${ }^{73}$.

Hacia el futuro, parece que el panorama es más alentador, pues el presupuesto plurianual para el período 2015-2018 refleja un paulatino aumento para el renglón "Educación, investigación, cultura y actividades recreativas", de tal forma que para el año 2016 se han asignado L. 7.972.797.360, representando un aumento del 87.5\%. Para el año 2017 se prevé un aumento del 104.7\% con relación al 2015 y del $9.1 \%$ con relación al año 2016, ascendiendo a L. 8.704.203.808. Para el 2018 el aumento previsto es del 107\% con relación al año 2015 y del 1.2\% con relación al año 2017, ascendiendo a L. 8.807.622.32674.

\section{Conclusiones}

A pesar que la Constitución de la República de Honduras no establece con meridiana taxatividad el derecho que los ciudadanos tienen a acceder, gozar y participar de la cultura, el control de convencionalidad al que ésta está sometida permite que, por la integración de los tratados internacionales que Honduras ha suscrito y ratificado, y por la aplicación del control de convencionalidad, éste quede plenamente reconocido. No obstante, es necesario que el legislador establezca sus alcances por medio de una norma secundaria.

Las obligaciones internacionales que el Estado de Honduras ha ido adquiriendo en esta materia son extensas, exigiendo que la estructura del Estado asuma compromisos con la sociedad misma, que no ha podido cumplir a cabalidad, pues el acceso a la cultura no es amplio ni masivo como los instrumentos internacionales lo sugieren.

Esto queda evidenciado en la transferencia de la responsabilidad que el Estado hace a la población en los instrumentos de planificación vigentes, desembarazándose de alguna manera de una responsabilidad que le es inherente, por ser parte del bienestar común que está llamado a garantizar. Pero es aún más evidente con el trato marginal que se le ha dado en la institucionalidad gubernamental en este período de gobierno, así como desde la perspectiva presupuestaria.

Ante este comportamiento quedan muchas dudas con relación a la intención que tiene el Estado de alcanzar las metas a mediano y largo plazo que se ha planteado, pues no parece estar enfocado en dedicar los recursos necesarios para

73 Secretaría de Finanzas (2015c), p. 1.

74 Secretaría de Finanzas (2015d), p. 1. 
cumplir con el compromiso que por medio de esos instrumentos de planificación ha adquirido con la ciudadanía.

\section{BibliografíA}

Carbonell, Miguel (2013): "Introducción general al control de convencionalidad”, en L. R. González Pérez, y D. Valadés, El constitucionalismo contemporáneo. Homenaje a Jorge Carpizo (México D. F., Instituto de Investigaciones Jurídicas), pp. 67-95.

Comisión Mundial de Ambiente y Desarrollo (1987): “Our common future”, [Fecha de consulta: 15 de julio de 2015]. [Disponible en: http://www.un-documents.net/our-common-future.pdf].

Cowan, Jane K. (2010): "Cultura y derechos después de Culture Rights”, en Revista de Antropología Social (No 19), pp. 67-101.

Dominici, Domenico (2005): "La tutela dei beni culturali. L'esperanza italiana”, en G. Ruiz-Rico Ruiz, y N. Pérez Sola, Constitución y cultura (Valencia, Tirant lo Blanch), pp. 159-170.

Donders, Yvone (2010): "Do cultural diversity and human rights make a good match?”, en International Social Science Journal (No 199), pp. 15-35.

Dorantes Díaz, Francisco Javier (2013): "Derecho a la cultura en México. $\mathrm{Su}$ constitucionalización, sus características, alcances y limitaciones", en Alegatos-Revista Jurídica de la Universidad Autónoma Metropolitana (No 85), pp. 845-862.

García Fernández, Javier (2005): "La función del Derecho en la protección de los bienes culturales", en G. Ruiz-Rico Ruiz, y N. Pérez Sola, Constitución y cultura (Valencia, Tirant lo Blanch), pp. 45-80.

GonZÁlez Moreno, Beatriz (2003): Estado de cultura, derechos culturales y libertad religiosa. $19^{\text {a }}$ ed. (Madrid, Civitas).

Mazuelos Bellido, Ángeles (2004): “Soft law: ¿Mucho ruido y pocas nueces?”, en Revista Electrónica de Estudios Internacionales (No 8), pp. 1-40.

Núñez Rivero, Cayetano y Pérez A., Carla Z. (2015): "La diversidad cultural como derecho fundamental de los pueblos indígenas", en Revista de Derecho UNED (No 17). Pp. 57-71.

Organización de Estados Americanos (1948): "Carta de la Organización de los Estados Americanos". [Fecha de consulta: 15 de julio de 2015]. [Disponible en: http://www.oas.org/dil/esp/tratados_A-41_Carta_de_la_Organizacion_ de_los_Estados_Americanos.pdf]. 
Organización de Estados Americanos (1969): "Convención Americana sobre Derechos Humanos". [Fecha de consulta: 15 de julio de 2015]. [Disponible en: http://www.oas.org/dil/esp/tratados_B-32_Convencion_Americana_sobre_Derechos_Humanos.pdf].

Organización de las Naciones Unidas (1948): "Declaración Universal de Derechos Humanos". [Fecha de consulta: 10 de agosto de 2016]. [Disponible en: http://www.un.org/es/documents/udhr/index_print.shtml].

Organización de las Naciones Unidas (1966): "Pacto Internacional de Derechos Económicos, Sociales y Culturales". [Fecha de consulta: 10 de julio de 2015]. [Disponible en: http://www.acnur.org/t3/fileadmin/scripts/doc.php?file=t3/ fileadmin/Documentos/BDL/2001/0014].

Organización de las Naciones Unidas (1977): "Resolución 32/130 de la Asamblea General de Naciones Unidas". [Fecha de consulta: 10 de julio de 2015]. [Disponible en: http://www.un.org/es/comun/docs/?symbol=A/ RES/32/130\&Lang=S].

Organización de las Naciones Unidas para la Educación, la Ciencia y la Cultura (1954): "Convención para la protección de la propiedad cultural en caso de conflicto armado". [Fecha de consulta: 1 de julio de 2015]. [Disponible en: http://portal.unesco.org/es/ev.php-URL_ID=13637\&URL_DO=DO_ TOPIC\&URL_SECTION=201.html].

Organización de las Naciones Unidas para la Educación, la Ciencia y la Cultura (1970): "Convención sobre las medidas que deben adoptarse para prohibir la importación, la exportación y la transferencia de propiedades ilícitas de bienes culturales". [Fecha de consulta: 1 de julio de 2015]. [Disponible en: http://portal.unesco.org/es/ev.php-URL_ID=13039\&URL_DO=DO_ TOPIC\&URL_SECTION=201.html].

Organización de las Naciones Unidas para la Educación, la Ciencia y la Cultura (1972): "Convención sobre la Protección del Patrimonio Mundial Cultural y Natural”. [Fecha de consulta: 1 de julio de 2015]. [Disponible en: http://whc.unesco.org/archive/convention-es.pdf].

Organización de las Naciones Unidas para la Educación, la Ciencia y la Cultura (1976 a): "Recomendación relativa a la participación y la contribución de las masas populares en la vida cultural". [Fecha de consulta: 1 de julio de 2015]. [Disponible en: http://portal.unesco.org/es/ev.phpURL_ID=13097\&URL_DO=DO_TOPIC\&URL_SECTION=201.html].

Organización de las Naciones Unidas para la Educación, la Ciencia y la Cultura (1976 b): "Recomendación sobre el intercambio internacional de 
bienes culturales". [Fecha de consulta: 1 de julio de 2015]. [Disponible en: http://portal.unesco.org/es/ev.php-URL_ID=13132\&URL_DO=DO_TOPIC\&URL_SECTION=201.html].

Organización de las Naciones Unidas para la Educación, la Ciencia y la Cultura (2001 a): "Convención sobre la Protección del Patrimonio Cultural Subacuático”. [Fecha de consulta: 1 de julio de 2015]. [Disponible en: http://portal.unesco.org/es/ev.php-URL_ID=13520\&URL_DO=DO_TOPIC\&URL_SECTION=201.html].

Organización de las Naciones Unidas para la Educación, la Ciencia y la Cultura (2001 b): "Declaración Universal de la Unesco sobre la Diversidad Cultural". [Fecha de consulta: 10 de julio de 2015]. [Disponible en: http:// portal.unesco.org/es/ev.php-URL_ID=13179\&URL_DO=DO_TOPIC\&URL_SECTION=201.html].

Organización de las Naciones Unidas para la Educación, la Ciencia y la Cultura (2003 a): "Convención sobre la Salvaguardia del Patrimonio Cultural Inmaterial”. [Fecha de consulta: 10 de julio de 2015]. [Disponible en: http:// unesdoc.unesco.org/images/0013/001325/132540s.pdf].

Organización de las Naciones Unidas para la Educación, la Ciencia y la Cultura (2003 b): "Declaración de la Unesco relativa a la destrucción intencional del patrimonio cultural”. [Fecha de consulta: 15 de julio de 2015]. [Disponible en: http://portal.unesco.org/es/ev.php-URL_ID=17718\&URL_ DO=DO_TOPIC\&URL_SECTION=201.html].

Organización de las Naciones Unidas para la Educación, la Ciencia y la Cultura (2005): “Convención sobre la Protección y Promoción de la Diversidad de Expresiones Culturales". [Fecha de consulta: 15 de julio de 2015]. [Disponible en: http://portal.unesco.org/es/ev.php-URL_ID=31038\&URL_ DO=DO_TOPIC\&URL_SECTION=201.html].

Pastor Ridruejo, José Antonio (2015): Curso de Derecho Internacional Público y de Organizaciones Internacionales. 19a ed. (Madrid, Editorial Tecnos).

Presidencia de la República (2014): "Plan Estratégico de Gobierno, 2014-2018”. [Fecha de consulta: 12 de julio de 2015]. [ Disponible en: http://www.sep. gob.hn/sitio/transparencia/descargas/2015/Plan_Estratégico_del_Gobierno_de_Honduras_2014-2018.pdf].

Picontó Novales, Teresa (1992): "Teoría general de la interpretación hermenéutica jurídica: Betti y Gadamer”, en Anuario de Filosofía del Derecho IX (No 9), pp. 223-248. 
República de Honduras (1982): “Decreto 131-1982, Constitución de la República de Honduras". [Fecha de consulta: 2 de julio de 2015]. [Disponible en: http://www.congresonacional.hn/transparencia/images/diariooficial/DecretoNo1311982ConstituciondelaRepublicadeHonduras.pdf].

República de Honduras (2010): "Visión de País 2010-2038 y Plan de Nación 2010-2022". [Fecha de consulta: 14 de julio de 2015]. [Disponible en: http:// www.se.gob.hn/media/files/leyes/LC_10.pdf].

República de Honduras (2013): “Decreto 266-2013, Ley para optimizar la administración pública, mejorar los servicios a la ciudadanía y fortalecimiento de la transparencia en el gobierno". [Fecha de consulta: 14 de julio de 2015]. [Disponible en: http://www.tsc.gob.hn/biblioteca/index.php/leyes/527-ley-para-optimizar-la-administracion-publica-mejorar-los-servicios-a-la-ciudadania-y-fortalecimiento-de-la-transparencia-en-el-gobierno].

República de Honduras (2014): “Decreto Ejecutivo PCM 078-2014”. [Fecha de consulta: 14 de julio de 2015]. [Disponible en: http://www.tzibalnaah.unah. edu.hn/bitstream/handle/123456789/963/20141229.pdf?sequence=2\&isAllowed $=y$ ].

Ruiz-Rico Ruiz, Gerardo (2005): "Introducción metodológica sobre el estudio de la cultura por el derecho constitucional”, en G. Ruiz-Rico Ruiz, y N. Pérez Sola, Constitución y cultura (Valencia, Tirant lo Blanch), pp. 9-34.

Secretaría de Estado en el Despacho Presidencial (2013): "Informe Preliminar de Avances del Plan de Gobierno Abierto Honduras 2013". [Fecha de consulta: 17 de julio de 2015]. [Disponible en: http://www.sep.gob.hn/sitio/images/ stories/transparenciafiscal/Informe_de_paln_de_Gobierno_Abierto.pdf].

Secretaría de Estado en el Despacho Presidencial (2014): "Memoria de Labores 2014". [Fecha de consulta: 17 de julio de 2015]. [Disponible en: http:// www.sep.gob.hn/sitio/transparencia/descargas/2015/Memoria\%20de\%20 Labores\%20SDP\%202014.pdf].

Secretaría de Finanzas (2015 a): "Presupuesto de Egresos por Finalidad y Función". [Fecha de consulta: 17 de julio de 2015]. [Disponible en: http://www. sefin.gob.hn/wp-content/uploads/Presupuesto/2015/aprobado/estadisticas/r_ fpr_cmp_fin_fun.pdf].

Secretaría de Finanzas (2015 b): "Presupuesto de Egresos por Institución y Finalidad”. [Fecha de consulta: 17 de julio de 2015]. [Disponible en: http://www.sefin.gob.hn/wp-content/uploads/Presupuesto/2015/aprobado/ estadisticas/r_fpr_ins_fina.pdf]. 
Secretaría de Finanzas (2015 c): "Presupuesto de Egresos por Programa de la Administración Central". [Fecha de consulta: 17 de julio de 2015]. [Disponible en: http://www.sefin.gob.hn/wp-content/uploads/Presupuesto/2015/ aprobado/estadisticas/r_fpr_cmp_prog.pdf].

Secretaría de Finanzas (2015 d): “Presupuesto Plurianual 2015-2018”. [Fecha de consulta: 17 de julio de 2015]. [Disponible en: http://www.sefin.gob.hn/ wp-content/uploads/Presupuesto/2015/aprobado/Descentralizadas/r_fpr_pluri_finfun_ac.pdf].

Secretaría de la Presidencia (2015): "Plan Operativo Anual 2015". [Fecha de consulta: 17 de julio de 2015]. [Disponible en: http://www.sep.gob.hn/sitio/ transparencia/descargas/2015/POA_SEP_2015.pdf].

\section{JURISPRUDENCIA CITADA}

Ramos Soto y otros con Congreso Nacional (2015): Sala de lo Constitucional de la Corte Suprema de Justicia de 22 de abril de 2015 (recurso de inconstitucionalidad) en: Sistema de Indexación Jurisprudencial. [Fecha de consulta: 11 de agosto de 2015]. [Disponible en: http://www.poderjudicial.gob.hn/ ConsultaHN/VerAmparo.aspx?reg=5445].

Feliú Corcuera y otros con Consejo de Dirección del Organismo Autónomo "Medios de Comunicación Social del Estado" (1981): Sala Segunda del Tribunal Supremo Constitucional de 16 de marzo de 1981 (recurso de amparo) en: Boletín Oficial del Estado, núm. 89 (1981), pp. 1-5. 